\title{
Direkte Demokratie im Rathaus: Wie bewerten die Kommunalverwaltungen in Nordrhein-Westfalen die Praxis?*
}

\author{
Jens Kösters
}

Elemente direkter Demokratie gehören mittlerweile zum Standardrepertoire der Kommunalverfassungen in Deutschland. Die Öffnung der Gemeindeordnungen zugunsten einer verstärkten unmittelbaren Bürgerbeteiligung war mitunter hart umkämpft, der Weg zum Ziel keineswegs vorgezeichnet. Dennoch haben sich die Partizipationsbefürworter mit dem Argument durchgesetzt, dass ein Bekenntnis zu ergänzenden Beteiligungsformen keinen Verrat am kommunalen Repräsentationsprinzip darstellt, sondern dass die demokratische Grundordnung insgesamt durch die behutsame Anreicherung um weitere Einflussmöglichkeiten an Profil zu gewinnen vermag.

Nach Baden-Württemberg (1956), Schleswig-Holstein und Sachsen-Anhalt (1990), den übrigen ostdeutschen Bundesländern Brandenburg, Mecklenburg-Vorpommern, Sachsen und Thüringen sowie Hessen (1993) und Rheinland-Pfalz (1994) hat sich auch der nordrhein-westfälische Gesetzgeber im Jahre 1994 dazu entschlossen, die Gemeindeordnung als Zentrum der Kommunalverfassung um direktdemokratische Handlungsoptionen zu erweitern. Entgegen einer weit verbreiteten Auffassung sind unter direkter Demokratie Entscheidungen über bestimmte Sachangelegenheiten zu subsumieren, nicht jedoch Entscheidungen, die die Personalauslese innerhalb einer Gemeinde betreffen. Bürgerbegehren und -entscheid sind demzufolge von der engen Definition erfasst, während die Direktwahl hauptamtlicher Bürgermeister (weiterhin) im Bereich der repräsentativen Demokratie zu verorten ist.

Der Versuch, nach inzwischen zehnjähriger Anwendungspraxis ${ }^{1}$ in Nordrhein-Westfalen ein umfassendes Erfahrungsbild über die Rechtsinstitute Bürgerbegehren und Bürgerentscheid nachzuzeichnen, fördert zweierlei zutage: zum einen, dass entsprechende Ausarbeitungen dünn gesät sind, und zum anderen, dass sich die meisten Studien auf einen Akzeptanztest bei jenen Akteuren beschränken, für die diese Beteiligungsrechte in erster Linie institutionalisiert worden sind. Während der Bürger als potenzieller „Kunde“ der angebotenen „Dienstleistung“ gern ausführlich wissenschaftlich befragt wird, liegen die Standpunkte der Städte und Gemeinden oftmals im Verborgenen ${ }^{2}$. Doch auch - und

* Dieser Text beruht in Teilen auf den Ergebnissen einer Studie, die sich unter anderem mit „Bürgerbegehren und Bürgerentscheid aus Sicht der Städte und Gemeinden“ beschäftigt hat (Fn. 3). Insbesondere ist auf das erhobene Datenmaterial der Primäruntersuchung zurückgegriffen worden. Die überarbeitete Auswertung und grundlegend geänderte Schwerpunktsetzung und Themenanordnung wird im Rahmen dieser Veröffentlichung zudem um eine eigenständige Schlussbewertung komplettiert. Mein besonderer Dank gilt jenen Städten und Gemeinden in Nordrhein-Westfalen, die aufgrund ihrer bereitwilligen Mithilfe das Zustandekommen dieses Beitrags überhaupt erst ermöglicht haben.

1 Vgl. Ralph Kampwirth, Der ernüchterte Souverän. Bilanz und Perspektiven der direkten Demokratie in den 16 Bundesländern und auf Kommunalebene, in: ZParl, 34. Jg. (2003), H. 4, S. 657 - 671, der hierzu bundesweit Stellung bezieht.

2 Eine löbliche Ausnahme ist beispielsweise Klaus Schulenburg, Plebiszitäre Elemente der neuen Gemeindeordnung in Nordrhein-Westfalen aus Sicht der Bürgermeister und Gemeindedirek- 
gerade - die Kommunalverwaltungen sind in diesen Evaluationsprozess sinnvollerweise einzubeziehen. Denn sie vermögen Aufschluss darüber zu geben, welche detaillierten Bewertungsmuster aus gemeindlicher Sicht vorherrschend sind. Aus diesem Grunde sollen nachfolgend die verwaltungsseitig vertretenen Positionen anhand eines standardisierten Fragebogens ermittelt werden ${ }^{3}$, nicht zuletzt, weil die Gemeinden zwar durch die Einbindung in kommunale Spitzenverbände (Städtetag, Städte- und Gemeindebund) ein Sprachrohr besitzen, welches jedoch nicht zwangsläufig deckungsgleiche Positionen vertritt, sondern in einer mitunter schwierigen Gratwanderung Bündelungsfunktionen wahrnehmen muss.

\section{Gesetzlicher Handlungsrahmen}

Bevor die Umfrageergebnisse dargestellt werden, sind wichtige Hinweise zum gesetzlichen Befragungsgegenstand vorauszuschicken. Mit einem Bürgerbegehren können die Bürger beantragen, dass sie an Stelle des Rates über eine Angelegenheit der Gemeinde entscheiden ${ }^{4}$. Das Verfahren besteht ausweislich dieser Legaldefinition aus einer Antragsund Durchführungsstufe. Damit es überhaupt zum Bürgerentscheid - also der eigentlichen Abstimmung, zu deren Teilnahme sämtliche Gemeindebürger aufgerufen sind kommen kann, muss ein Bürgerbegehren vom Gemeinderat für zulässig erklärt werden. Die Zulässigkeit wiederum hängt von bestimmten, vornehmlich im Gesetz formulierten Voraussetzungen ab, die sowohl formeller als auch materieller Natur sind ${ }^{5}$.

So muss ein Bürgerbegehren einen nach den gesetzlichen Bestimmungen durchführbaren Vorschlag für die Deckung der Kosten der verlangten Maßnahme enthalten $(\$ 26$ Abs. 2 GO NW) und - sofern es sich gegen einen Beschluss des Rates wendet - innerhalb einer Frist von drei Monaten respektive sechs Wochen bei bekanntmachungspflichtigen Beschlüssen eingereicht werden (Abs. 3). Außerdem ist eine ausreichende Anzahl von Unterstützungsunterschriften beizubringen, deren Höhe sich an der Einwohnerzahl orientiert und mit zunehmender Größe abnimmt (Abs. 4). Aus materieller Sicht listet ein Negativkatalog bestimmte kommunale Politikbereiche und -gegenstände auf, die einem Bürgerbegehren von vornherein entzogen sind (Abs. 5) .

Auf dieser Grundlage hat der Gemeinderat anschließend die (Un-)Zulässigkeit eines zuvor schriftlich eingereichten Antrages festzustellen (Abs. 6). Hierbei kommt dem Rat kein politischer Beurteilungs- oder Ermessensspielraum zu. Ist das Begehren zulässig,

toren, in: ZParl, 32. Jg. (2001), H. 1, S. 72 - 93. Ferner - bezogen auf die Kreisebene - ebenfalls Klaus Schulenburg, Die Kommunalpolitik in den Kreisen Nordrhein-Westfalens: eine empirische Bestandsaufnahme, Köln / Stuttgart 2001.

3 Vgl. Jens Kösters, Der Bürgerentscheid in Nordrhein-Westfalen. Politische Ausgestaltung und Rechtsetzung der Gemeinden, Reihe: Politik und Partizipation, Band 4, Münster 2005, S. 151 $-201$.

$4 \$ 26$ Abs. 1 der Gemeindeordnung für das Land Nordrhein-Westfalen (GO NW).

5 Vgl. Innenministerium des Landes Nordrhein-Westfalen (Hrsg.), Bürgerbegehren und Bürgerentscheid - Leitfaden, 6. Auflage, 2005, als praktische Anleitungshilfe.

6 Einen vollständigen Überblick zum Negativkatalog bietet Klaus Ritgen, Bürgerbegehren und Bürgerentscheid: Dargestellt am Beispiel des $\$ 26$ der nordrhein-westfälischen Gemeindeordnung, Baden-Baden 1997, S. $184-213$. 
steht es dem Rat allerdings politisch völlig frei, ob er den erhobenen Forderungen einer Initiative von sich aus stattgeben will oder ob er eine Entscheidung im Rahmen eines Bürgerentscheids bevorzugt. Im letztgenannten Fall muss die zur Abstimmung gestellte Frage nicht nur von der Mehrheit der gültigen Stimmen getragen werden, sondern zugleich mindestens 20 Prozent aller Gemeindebürger ausmachen, um Verpflichtungskraft beanspruchen zu können (Abs. 7). Auf das Zulassungsquorum beim Bürgerbegehren folgt somit ein Zustimmungsquorum beim Bürgerentscheid. ${ }^{7}$ Sofern der Bürgerentscheid erfolgreich ist, kann er vor Ablauf einer zweijährigen Bindungswirkung lediglich auf Initiative des Rates durch ein neues Referendum abgeändert werden (Abs. 8). Was die konkreten Durchführungsmodalitäten etwa im Hinblick auf die Stimmabgabe per Brief oder eine persönliche Benachrichtigungskarte anbelangt, ist das Innenministerium zur Regelung mittels Rechtsverordnung ermächtigt (Abs. 10) ${ }^{8}$.

Ein Großteil der skizzierten Bedingungen ist zumindest im Kern bundesweit identisch - jedenfalls was den groben Verfahrensablauf betrifft; gleichwohl variiert der gesetzliche Anforderungsgrad in spezifischen Bereichen in durchaus beachtlicher Weise? ${ }^{9}$ Hierzu gehört beispielsweise, dass es in Nordrhein-Westfalen keine von Gesetzes wegen garantierte aufschiebende Wirkung gibt, mittels derer es der Gemeinde untersagt ist, dem Begehren durch wie auch immer geartete Maßnahmen kurzerhand die Grundlage zu entziehen. Allerdings ist dem Rat auch die Möglichkeit verwehrt, selbst an das Gemeindevolk heranzutreten, um eine Angelegenheit dem Wählervotum zu unterstellen ${ }^{10}$.

7 Zur Problematik von Zustimmungsquoren vgl. Thorsten Hüller, Herrschaft des Quorums? Ein Vorschlag zur Lösung eines Problems direkter Demokratie, in: ZParl, 37. Jg. (2006), H. 4, S. $823 \mathrm{ff}$.

8 Vgl. Jens Kösters, a.a.O., S. 69 ff. Der ursprüngliche Gesetzentwurf der Landesregierung sah den Erlass einer Rechtsverordnung zwingend vor; kurz vor der abschließenden Beschlussfassung im Landtag wurde diese Vorlage jedoch insofern modifiziert, als - auf Antrag der SPDMehrheitsfraktion - statt eines verpflichtenden nur ein fakultativer Erlass unter Billigung aller Fraktionen festgeschrieben wurde. Hintergrund war, es den Gemeinden zunächst selbst zu überlassen, das $\mathrm{Ob}$ und Wie entsprechender Regelungen zu bestimmen. Grundsätzlich bestehen folgende Möglichkeiten zur Durchführung eines Bürgerentscheides: Die analoge Anwendung des Kommunalwahlgesetzes, der Erlass einer ministeriellen Ausführungsverordnung sowie das in das Ermessen der jeweiligen Gemeinde gestellte Verfahren.

9 Vgl. Ralph Kampwirth, a.a.O., S. 664. Außerdem Hans Herbert von Arnim, Vom schönen Schein der Demokratie. Politik ohne Verantwortung - am Volk vorbei, München 2001, S. 210.

10 Trotz dieser diffusen, je nach Bundesland schwankenden Ausgangslage, die auf die Gesetzgebungskompetenz der Länder im Bereich der Kommunalpolitik zurückzuführen ist, erscheint es nicht zwingend geboten, die zu evaluierenden Elemente zunächst einem umfassenden Ländervergleich zu unterziehen, zumal das Augenmerk prinzipiell auf Nordrhein-Westfalen gerichtet ist. Vgl. Philipp Karr, Institutionen direkter Demokratie in den Gemeinden Deutschlands und der Schweiz. Eine rechtsvergleichende Untersuchung, Rostocker Arbeiten zum Internationalen Recht, Band 8, Baden-Baden 2003, dessen Dissertation die mitunter komplexe Materie unter sauberer Trennung der inhaltlichen Bestandteile eines Bürgerbegehrens und -entscheides analysiert. 


\section{Konzeption, Rücklaufquote und Datenaufschlüsselung}

Die Umfragedaten sind im Rahmen einer wissenschaftlichen Untersuchung zur Evaluierung von Bürgerbegehren und Bürgerentscheid erhoben worden ${ }^{11}$. Eine vertrauliche Behandlung der abgefragten Meinungen und Standpunkte wurde persönlich garantiert. Da eine möglichst flächendeckende Teilnahme angestrebt war, sollten die Kommunen ungeachtet eventueller Praxiserfahrungen zur Beantwortung ermutigt werden. Dieses Vorgehen begegnet deshalb keinen Bedenken, weil die Gemeinden mit den in $₫ 26$ GO NW normierten Rechtsinstituten grundsätzlich vertraut sind. Insoweit ist es ratsam, den Wissensvorrat der kommunalen Selbstverwaltung, die zur qualifizierten Bewertung fähig ist, voll auszuschöpfen. Der Fragenkatalog bedient sich größtenteils geschlossener Fragen in Form der Antwortalternativen „ja“, „nein“ und „weiß nicht“. Das insoweit eingeschränkte Antwortspektrum wurde allerdings dadurch aufgelockert, dass eine individuelle Begründung, Ergänzung oder Kommentierung nicht nur möglich, sondern ausdrücklich erwünscht war. Weiterhin orientiert sich der Gliederungsansatz des Fragenkatalogs an dem Gerüst der Gesetzesgrundlage; die Tatbestandsmerkmale werden folglich schrittweise abgearbeitet.

Der Befragungszeitraum erstreckte sich von April bis einschließlich August 2004; die vierseitigen Fragebögen sind an sämtliche Städte und Gemeinden per E-Mail mit der Bitte versandt worden, diese postalisch zurückzuschicken. Für eine Befragung, die sich zur Kontaktaufnahme ausschließlich auf die elektronischen Medien verlässt, ist die Rücklaufquote von 85,4 Prozent (das sind 338 von 396 Kommunen) umso erstaunlicher. Die sehr hohe Beteiligung resultiert nicht nur daraus, dass der Fragebogen offensichtlich als Offerte verstanden wurde, Meinungen und Interessen freimütig zu artikulieren, sondern auch aus der „online“-Affinität der Verwaltungen. Eine Kontaktaufnahme per E-Mail erweist sich also keineswegs als Zugangsbarriere. Zum Beantwortungskreis ist anzumerken, dass die gemeindliche Führungsspitze ${ }^{12}$ 78,5 Prozent aller eingegangenen Fragebögen abbildet.

Nicht zuletzt aufgrund der unerwartet hohen Rücklaufquote des Fragebogens empfiehlt es sich, die erhobenen Daten über die Darstellung in absoluten Zahlen hinaus zusätzlich nach gesonderten Kriterien aufzuschlüsseln. Als zentrale Bezugskonstante fungiert daher die Größenstruktur der kommunalen Gebietskörperschaften, welche eine vierfache Abstufung enthält: Neben die 23 kreisfreien Städte treten in Nordrhein-Westfalen 35 Große kreisangehörige Städte mit mehr als 60.000 Einwohnern, 119 Mittlere kreisangehörige Städte mit mehr als 25.000 (aber weniger als 60.000) Einwohnern sowie 219 Städte und Gemeinden bis zu einer Einwohnerzahl von 25.000. Soweit erforderlich, wird zudem auf die Zuordnung zu den fünf Regierungsbezirken mit Sitz in Arnsberg, Detmold, Düsseldorf, Köln und Münster rekurriert, die als staatliche Mittelinstanzen eine Scharnierfunktion zwischen Land und Kommunen ausüben und Interpretationshinweise für eine eher ländliche oder urbane Prägung liefern können. Eine eventuelle Vorbelastung einzelner Gemeinden durch bereits absolvierte Bürgerentscheide ist bei geeigneter Fragestellung ebenfalls in der Auswertung berücksichtigt.

11 So auch der Titel des Fragebogens, vgl. Jens Kösters, a.a.O., S. 151.

12 Das sind Bürgermeister, Allgemeine Vertreter, Beigeordnete, Amts- und Fachbereichsleiter, persönliche Referenten. 


\section{Ergebnisse: Bürgerbegehren und Bürgerentscheid aus Sicht der Kommunalverwaltungen}

\subsection{Rechtlicher Rahmen}

Die im Jahre 2000 nachträglich in die Gemeindeordnung eingefügte Passage, wonach die Verwaltung „in den Grenzen ihrer Verwaltungskraft ihren Bürgern bei der Einleitung eines Bürgerbegehrens behilflich" ist ( $\$ 26$ Abs. 2 Satz 3 GO NW), genießt einen deutlichen Rückhalt bei denjenigen, die dies unter Umständen zu leisten haben (vgl. Tabelle 1). Nur jede vierte Gemeinde lehnt eine solche Beratungspflicht bei vereinzelten Enthaltungen ab. Dieses sehr deutliche, in Zeiten eines zunehmenden Arbeits- und Aufgabenvolumens überraschende Votum erfährt sogar noch eine Steigerung, weil die ablehnenden Haltungen teilweise der Relativierung bedürfen. Von 76 zusätzlich abgegebenen Kommentaren ergreifen 46 für und 28 gegen die Hilfestellung Partei; letztgenannte Meinungsbekundungen sind jedoch zur Hälfte nicht per se gegen eine Beratungsverpflichtung gerichtet, sondern verweisen vielmehr - was inhaltlich zutrifft - darauf, dass bereits eine allgemeine gesetzliche Beratungspflicht bestehe ( $\$ 22$ Abs. 1 GO NW) oder dass die Hilfeleistung im Zeitalter der forcierten Kundenorientierung ohnehin eine Selbstverständlichkeit darstelle, die nicht gesondert betont werden müsse. Rechnet man diese Auswertung unter Berücksichtigung des Spezialisierungsschubes hoch, kann gar von einer Gesamtzustimmung von über 80 Prozent zugunsten der Beratungspflicht ausgegangen werden.

Aus kommunaler Sicht haben sich des Weiteren die Einreichungsfristen für kassierende Bürgerbegehren bewährt, die zwischen bekanntmachungspflichtigen und -freien Beschlüssen unterscheiden ${ }^{13}$. Jene 15 Gemeinden, die eine Änderungsbedürftigkeit bejahen, fordern zu 60 Prozent eine Herabsetzung der Fristen, was de facto eine Verschärfung bedeutet, da die Unterschriften insoweit binnen verkürzter Frist gesammelt werden müssten. Das im Ländervergleich einmalige Kombinationsmodell, welches sich an der Bekanntmachungsform ausrichtet, wird damit nicht infrage gestellt, so dass nach gemeindlicher Auffassung vorerst kein Weg an der durchaus ungewöhnlichen Aufteilung vorbeiführt.

Geradezu überwältigend fällt das Votum im Hinblick auf den Negativkatalog aus, dessen grundsätzliche Existenz für unverzichtbar gehalten wird. Die mitunter in den wissenschaftlichen Fachdisziplinen vertretene Auffassung, dass jede Gemeindeangelegenheit ein thematisierungsfähiger Gegenstand eines Bürgerbegehrens sein sollte, wird verwaltungsseitig mit einer nicht zu überbietenden Eindeutigkeit abgelehnt. Auch der Weiterentwicklung des Negativkatalogs - gleichgültig ob in Richtung einer Verlängerung oder Verkürzung der Ausschlussliste - wird eine deutliche Absage erteilt. Partiell unzufriedene Gemeinden regen jedoch einerseits eine Erweiterung des Negativkatalogs an, die letztlich auf gemeindliche Finanzaspekte zuläuft, und sie signalisieren andererseits eine wenn auch sehr schmale Bereitschaft, den Kranz zulässiger Themen womöglich um den Bereich der für den Bürger attraktiven Bauleitplanung zu erweitern. Dennoch kann diese Verdich-

13 Für Beschlüsse, die der Bekanntmachung bedürfen, gelten die Vorschriften der Bekanntmachungsverordnung vom 26. August 1999 (GV.NW. 1999, S. 516), vgl. Innenministerium des Landes Nordrhein-Westfalen, a.a.O., S. 11. 
tung nicht darüber hinwegtäuschen, dass eine übergroße Mehrheit der Kommunen weiterhin auf den geltenden Ausschlussbestand fixiert ist.

Sehr intensiv und bisweilen unsachlich ist in der Vergangenheit die Diskussion geführt worden, ob es überhaupt zweckmäßig ist, dem Gemeinderat als potenziellen Gegenspieler bürgerschaftlichen Engagements die Kompetenz zur Feststellung der (Un-)Zulässigkeit eines Bürgerbegehrens anzutragen, da zu erwarten sei, dass dieser sachwidrige Überlegungen anstelle und die Entscheidung nicht auf ausschließlich rechtliche, sondern politische Argumente stütze ${ }^{14}$. Ungeachtet solcher abstrakter Gefahrenprognosen halten die Gemeinden an dem tradierten Zuordnungsgefüge fest und verneinen daher ein missbräuchliches Verhalten.

Die durch einen Bürgerentscheid im Erfolgsfall ausgelöste zweijährige Abänderungssperre, die nur auf Veranlassung des Rates durch einen erneuten Entscheid durchbrochen werden kann, wird ebenfalls ohne erkennbare Vorbehalte gebilligt. Damit wird die Dominanz der kommunalen Repräsentativstruktur weder abqualifiziert noch in ihr Gegenteil verkehrt; vielmehr wird von den Gemeinden selbst anerkannt, dass es einer Bindungswirkung bedarf, deren zeitliche Länge gut austariert zu sein scheint. Obgleich es hätte vermutet werden können, wird ein fehlendes Selbsteintrittsrecht der Vertretungskörperschaft innerhalb des zweijährigen Zeitfensters also nicht unbedingt beklagt.

\begin{tabular}{|c|c|c|c|c|c|c|}
\hline & \multicolumn{2}{|c|}{ ja } & \multicolumn{2}{|c|}{ nein } & \multicolumn{2}{|c|}{ weiß nicht } \\
\hline & $\mathrm{n}$ & $\%$ & $\mathrm{n}$ & $\%$ & $\mathrm{n}$ & $\%$ \\
\hline Notwendigkeit der Beratungspflicht & 238 & 70,6 & 86 & 25,5 & 13 & 3,9 \\
\hline Änderungsbedarf bei den Einreichungsfristen & 15 & 4,5 & 315 & 93,7 & 6 & 1,8 \\
\hline Notwendigkeit des Negativkatalogs & 335 & 99,4 & - & 0 & 2 & 0,6 \\
\hline Änderungsbedarf beim Negativkatalog & 24 & 7,2 & 295 & 88,9 & 13 & 3,9 \\
\hline Zulässigkeitsfeststellung dem Rat entziehen & 52 & 15,9 & 261 & 79,8 & 14 & 4,3 \\
\hline Änderungsbedarf bei der Abänderungssperre & 19 & 5,7 & 300 & 89,5 & 16 & 4,8 \\
\hline
\end{tabular}

Schlüsselt man die Daten entsprechend der Einwohnergröße der befragten Kommunen auf, so kommen in Bezug auf sämtliche Fragen - Beratungspflicht, Einreichungsfristen, Negativkatalog, Kompetenzzuweisung an den Gemeinderat beim Zulässigkeitsbeschluss und Abänderungssperre - beinahe deckungsgleiche Prozentwerte zum Vorschein. Leichte Schwankungen sind allenfalls einer eher hohen oder niedrigen Fallzahl geschuldet und lassen sich nicht für gewagte Interpretationen heranziehen. Insofern sind die zum Ausdruck kommenden Mehrheitsmeinungen für alle Einwohnerklassen gleichermaßen tragbar, was für eine homogene Politikwahrnehmung spricht. Dennoch lassen sich gewisse Ungleichgewichte feststellen: Während 19 von 69 Kommunen im Regierungsbezirk Münster dafür plädieren (27,5 Prozent), dass dem Rat die Entscheidung über die Zulässigkeit eines Begehrens entzogen werden soll, schließen sich dem nur 5,8 Prozent der

14 Vgl. Fritz Ossenbühl, Bürgerbegehren und Bürgerentscheid, in: Gerhard Seiler (Hrsg.), Gelebte Demokratie, Festschrift für Manfred Rommel, Stuttgart 1997, S. 251 f. 
Kommunen im Regierungsbezirk Arnsberg an, wo die Akzeptanz der jetzigen Regelung mit über 90 Prozent am größten ist (im Münsteraner Bezirk 69 Prozent). Stellt man auf jene Kommunen ab, die bereits mindestens einen Bürgerentscheid absolviert haben und damit zwangsläufig eine Zulässigkeitsentscheidung treffen mussten, die jedoch zu keinem Übernahmebeschluss führte, so ergeben sich keine Auffälligkeiten; die Ablehnungsquote liegt hier bei knapp 17 Prozent, woraus geschlossen werden darf, dass sich in diesen Praxis erprobten Gemeinden kein anderes oder intensiveres Problembewusstsein entwickelt hat als in solchen Gemeinden, die überwiegend unter theoretischen Gesichtspunkten mit der Fragestellung befasst waren. ${ }^{15}$

Komplizierter gestaltet sich die Ausgangslage beim Kostendeckungsvorschlag. Hier kristallisiert sich mit absoluter Mehrheit (63,8 Prozent) der Wunsch nach einer Präzisierung heraus. Nur jede dritte Gemeinde (35,0 Prozent) hält an der jetzigen Formulierung fest; ein ersatzloser Verzicht findet keinerlei Rückhalt (0,6 Prozent), obgleich dies die bayerische Gemeindeordnung vormacht. Die dauerhafte Krise der Kommunalfinanzen wirkt hier insofern als Negativfolie, als die Gestaltungsspielräume äußerst eng sind. Vor diesem Hintergrund vertreten die Gemeinden mehrheitlich die Auffassung, dass an Initiativen, die aus den Reihen der Bürgerschaft an die Gemeinde herangetragen werden, genau dieselben Maßstäbe angelegt werden müssen, wie sie für den Rat schon längst gelten. Den größten Rückhalt dafür, den Kostendeckungsvorschlag unverändert zu lassen, findet sich bei den Gemeinden bis 25.000 Einwohner mit knapp 40 Prozent; demgegenüber meinen lediglich 28,6 Prozent der kreisfreien Städte, dass ihrer Einschätzung zufolge keine Veränderungen angezeigt sind.

\subsection{Zulassungs- und Zustimmungsquoren}

Da jedes Bürgerbegehren und jeder Bürgerentscheid von einer hinreichenden Anzahl an Gemeindebürgern getragen sein muss, ist sowohl ein Zulassungs- als auch ein Zustimmungsquorum installiert worden. So wird beim Bürgerbegehren eine an der jeweiligen Einwohnerzahl ausgerichtete Unterstützungsquote von mindestens drei und höchstens zehn Prozent der Bürger verlangt. Der Staffelungsgedanke trägt dem Umstand Rechnung, dass die Unterschriftensammlung in großen Städten schwieriger ist. Beim Bürgerentscheid wird demgegenüber ein von der Gemeindegröße unabhängiges Quorum von 20 Prozent verlangt, das nicht selten als „Stolperstein" ${ }^{16}$ wirkt. Diese Taxierungen vorausgeschickt, haben sich aus kommunaler Sicht sowohl das Zulassungs- als auch das Zustimmungsquorum bewährt (vgl. Tabelle 2), wobei die Zufriedenheit mit den unterschiedlich bemessenen Hürden beim Bürgerbegehren um rund zwölf Prozentpunkte ausgeprägter ist. Drei von vier Gemeinden beurteilen die jeweilige Höhe des Zulassungsquorums als "genau richtig“, nur jede fünfte ist der Meinung, diese sei „zu niedrig“. Auffällig ist, dass der Anteil jener Befragten, die von zu niedrigen Hürden sprechen, bei den kreisfreien

15 Ausführlicher - unter Einbeziehung eines speziell entwickelten Kriterienrasters, das ein Qualifikationsprofil verschiedener, zumindest vom Grundsatz her geeigneter Institutionen erstellt (Gemeinderat, Bezirksregierung, Innenministerium, Verwaltungsgericht) - Jens Kösters, a.a.O., S. $167-170$.

16 Ralph Kampwirth, a.a.O., S. 666. 


\begin{tabular}{|l}
\hline $\begin{array}{l}\text { Tabelle 2: Bewertung des Zulassungs-und Zustimmungsquorums in der nordrhein-westfälischen } \\
\text { Gemeindeordnung durch die Kommunalverwaltungen }\end{array}$ \\
\hline \\
\hline
\end{tabular}

Städten mit 31,8 Prozent signifikant über dem Wert der Kommunen bis 25.000 Einwohner liegt (19,9 Prozent), was nachvollziehbar ist, weil hier die mit drei bis fünf Prozent vermeintlich geringsten Anforderungen an den Erfolg eines Bürgerbegehrens gestellt werden. Die Staffelung wird also insbesondere in den Großstädten als ungerechtfertigter Nachteil empfunden. Dass die Abstufung primär eine gesetzgeberische Reaktion auf die mit zunehmender Einwohnergröße schwerfälligere Mobilisierung ist ${ }^{17}$, wird nicht überall anerkannt. Gleichwohl bleibt eine allgemein große Akzeptanz zur an der Gemeindegröße orientierten Abschichtung zu konstatieren, die als sachgerechte Lösung erachtet wird.

Beim Zustimmungsquorum werden ähnliche Tendenzen sichtbar, obwohl hier ein starrer Wert von 20 Prozent der Gemeindebürger statuiert ist. Während 32 Prozent der Kommunen bis 25.000 Einwohner der Auffassung sind, dass das Zustimmungsquorum zu niedrig angesetzt sei, sind dies bei den kreisfreien Städten schon 52 Prozent. Damit ist das Quorum ein aus kommunaler Sicht unverzichtbares Regulativ; eine deutliche Mehrheit erachtet die gegenwärtige Höhe für angemessen, immerhin jeder dritte Befragte hält allerdings eine Legitimationsverdichtung für zwingend geboten ${ }^{18}$.

Setzt man das Zustimmungsquorum in Abhängigkeit zum Zulassungsquorum, zeigen sich interessante Ergebnisse: Jene Gemeinden, die bereits das Zulassungsquorum als zu niedrig empfinden, sind zu 75 Prozent der Ansicht, dass dies auch für das Zustimmungsquorum gilt. Hier wird die durchgehende Unzufriedenheit mit beiden Quoren greifbar. Komplettiert werden diese Eindrücke dadurch, dass keine einzige Gemeinde, die das Zulassungsquorum für zu niedrig hält, im Gegenzug von einem zu hohen Zustimmungsquorum spricht. Von jenen Kommunen, die keine Bedenken gegenüber dem Zulassungsquorum geltend gemacht haben, will die übergroße Mehrheit von 77 Prozent beim Zustimmungsquorum ebenfalls nicht für Änderungen plädieren, woraus wiederum eine strukturelle Mehrheit zugunsten der jetzigen Regelung ablesbar ist.

Resümierend können daher zwei konkurrierende Grundmuster im Hinblick auf die Beurteilung der Quoren skizziert werden: Die herrschende Ansicht zeigt sich mit beiden Hürden einverstanden, eine qualifizierte Mindermeinung ist mit beiden unzufrieden. Die Fokussierung auf jene Kommunen, die bereits einen Bürgerentscheid durchgeführt haben und somit direkt mit dem Zustimmungsquorum konfrontiert waren, erhärtet die

17 Vgl. Hans Herbert von Arnim, a.a.O., S. 214; Philipp Karr, a.a.O., S. 165.

18 Obwohl das Zulassungsquorum degressiv gestaltet ist und am Höchstpunkt gerade einmal die Hälfte desjenigen Wertes beträgt, der für sämtliche Kommunen beim Zustimmungsquorum verbindlich ist, gibt es sich in der gemeindlichen Wahrnehmung als weniger akut zu erkennen. Oder anders gewendet: Trotz bedeutend höherer Hürden ist die Unzufriedenheit mit dem Zustimmungsquorum ausgeprägter, sodass dieses Kriterium als ungleich wichtiger eingestuft wird, zumal Erfolg und Scheitern eines Bürgerentscheids letztlich sehr stark von ihm abhängen. 
bislang getroffenen Aussagen insofern, als - wenn überhaupt - der Änderungsbedarf in erster Linie beim Zustimmungsquorum gesehen wird.

\subsection{Aufschiebende Wirkung, Terminierung und Ratsbürgerentscheid}

Mit der Frage nach der Notwendigkeit einer aufschiebenden Wirkung beim Bürgerbegehren wird erstmals der Regelungskorpus der Gesetzesgrundlage verlassen. Dadurch ist vermutlich zu erklären, dass die Einführung eines solchen Suspensiveffekts einerseits von einer Mehrheit der Befragten abgelehnt und andererseits von immerhin 24 Gemeinden nicht abschließend bewertet wird (vgl. Tabelle 3). Die kreisfreien Städte formulieren ihren Widerstand wiederum deutlicher, indem sie den Rechtsgedanken einer aufschiebenden Wirkung sogar zu 77 Prozent zurückweisen. Den größten Rückhalt findet der Suspensiveffekt im Regierungsbezirk Detmold mit 44,6 Prozent.

Aus der Zusammenschau der zusätzlich verfassten Kommentare gehen drei Meinungsrichtungen hervor. Ein erster Block bejaht die aufschiebende Wirkung vorbehaltlos, weil ein Bürgerbegehren nicht vor vollendete Tatsachen gestellt werden dürfe. Die Gegenansicht spricht insoweit von einer Blockade und Lähmung der Gemeindetätigkeit (passive Sichtweise) oder stellt heraus, dass jederzeit die Handlungs- und Entscheidungsfreiheit des Gemeinderates bestehen müsse (aktive Sichtweise). Eine eher vermittelnde Position arbeitet schließlich Bedingungen heraus, unter denen die Verankerung eines Suspensiveffektes aus rechtspolitischer Sicht wünschenswert erscheint ${ }^{19}$, wobei die Argumentationsstränge zwischen einem formell-rechtlichen und materiell-rechtlichen Ansatzpunkt differenzieren ${ }^{20}$.

Eine umgekehrte Ausgangslage hält die Frage nach der Zusammenlegung eines Bürgerentscheids mit einer allgemeinen Wahl bereit (vgl. Tabelle 3). Sechs von zehn Gemeinden plädieren für eine terminliche Kopplung, wobei die Zustimmungsraten bezüglich der Einwohnerstruktur über Kreuz liegen: Während rund 60 Prozent der Kommunen bis 60.000 Einwohner die Zusammenlegung befürworten, lehnen dies sowohl die Großen kreisangehörigen als auch die kreisfreien Städte mittels umgekehrter Mehrheit ab. Das Einvernehmen, Abstimmung und Wahl gemeinsam abzuwickeln, kippt demzufolge erstaunlicherweise bei Städten mit mehr als 60.000 Einwohnern. Dieser Auszählbefund wird im Übrigen dadurch empirisch bestätigt, dass im Regierungsbezirk Düsseldorf nur 40 Prozent der Kommunen für eine Zusammenlegung plädieren, während alle anderen eher ländlich geprägten Regierungsbezirke diese Minderheitenposition um jeweils 20 bis 30 Prozentpunkte übertreffen.

19 Vgl. auch Fritz Ossenbühl, a.a.O., S. 264, der sich - unabhängig von rechtlichen Erwägungen schon aus Gründen der politischen Klugheit dafür einsetzt, den Ausgang eines Bürgerbegehrens abzuwarten. Ausführlich und argumentativ überzeugend hierzu Philipp Karr, a.a.O., S. $178-184$.

20 Während der formelle Aspekt darauf zielt, die aufschiebende Wirkung erst nach Erreichen des Zulassungsquorums respektive nach Feststellung der Zulässigkeit des Bürgerbegehrens greifen $\mathrm{zu}$ lassen, wird unter materiellen Gesichtspunkten die Einschränkung diskutiert, dass kein unverhältnismäßiger Nachteil für die Gemeinde oder gar wirtschaftlicher Schaden eintreten dürfe. 
Zur Aufhellung der Motivlage tragen erneut die Kommentierungen bei. Für eine Zusammenlegung sprechen demnach drei Hauptargumente: erstens Kosten, zweitens organisatorische Gründe, die mit den Begriffen Verwaltungsaufwand und -effizienz umschrieben werden können, sowie drittens Partizipationsaspekte, die in eine gewünschte höhere Abstimmungsbeteiligung münden. Bei der Gegenansicht dominieren ebenfalls drei Motive: erstens Inkompatibilitätsgesichtspunkte, worunter Schlagworte wie Interferenzen, Irritationen und unzulässige Themenverquickung zu subsumieren sind, zweitens die Vermeidung von Mitnahmeeffekten respektive Betonung des Eigenständigkeitscharakters direktdemokratischer Verfahren sowie drittens die Missbrauchsgefahr, die aufgrund der Parteipolitisierung gegeben sei. Die Gemeinden lassen demnach keinen Zweifel daran aufkommen, dass im Falle einer gemeinsamen Absolvierung von Bürgerentscheid und Wahl neben erheblichen finanziellen Einsparungen infolge des „Huckepackeffekts “21 tatsächlich auch höhere Beteiligungsquoten erzielt werden. Unterschiedliche Auffassungen greifen erst hinsichtlich der Berechtigung eines solchen Effektes Platz: Während die herrschende Meinung die Gleichung aufstellt, wonach eine höhere Beteiligung am Bürgerentscheid eine höhere Legitimation der zur Abstimmung gestellten Sachfrage herzustellen vermag und allein schon deswegen anzustreben ist, blendet die Mindermeinung dieses Argument bewusst aus und hebt dagegen die Missbrauchsgefahr einer unheilvollen Vermengung grundverschiedener Politikmaterien hervor. Abschließend fördert die getrennte Darstellung der Kommunen mit respektive ohne Bürgerentscheid zutage, dass solche Gemeinden, die bereits ein Referendum absolviert haben, lediglich zur Hälfte der Ansicht sind, dass eine Zusammenlegung sinnvoll ist ${ }^{22}$.

Mit überaus deutlicher Mehrheit wird schließlich der so genannte Ratsbürgerentscheid $^{23}$ - auch bekannt als Ratsbegehren oder Ratsreferendum - abgelehnt (vgl. Tabelle 3). Lediglich jeder vierte Befragte kann diesem vom Gemeinderat initiierten Entscheid etwas Positives abgewinnen, wobei die Großen kreisangehörigen Städte mit 29,2 Prozent überrepräsentiert sind. Starke Schwankungen ergeben sich dann, wenn anstelle der Einwohnerzahl auf die Regierungsbezirke abgestellt wird. Während Arnsberg dem landesweiten Ergebnis fast exakt entspricht (77,1 zu 19,9 Prozent), driften Detmold (68,4 zu 26,3 Prozent) und Düsseldorf (89,3 zu 8,9 Prozent) weit voneinander ab. Weil die Aufschlüsselung nach Gemeindegrößenklassen zuvor keine aussagekräftige Tendenz zugelassen hat, ob der Widerstand zum Ratsbürgerentscheid mit steigender Einwohnerzahl eher zu- oder

21 Hans Herbert von Arnim, a.a.O., S. 228.

22 Dieses Ergebnis lässt mehrere Schlüsse zu. Zunächst könnte die geringere Zustimmung ein Indiz dafür sein, weshalb bislang - wohlgemerkt ungeachtet der sich praktisch ohnehin äußerst selten bietenden Gelegenheit - tatsächlich nur in den wenigsten Gemeinden eine Zusammenlegung erfolgt ist. Ferner könnte gemutmaßt werden, dass die vergleichsweise hohe Zustimmungsrate der Gemeinden ohne Bürgerentscheid spätestens dann spürbar absinkt, wenn der viel zitierte Ernstfall eintritt, das heißt wenn ein kassatorischer Bürgerentscheid durchzuführen ist.

23 Mit einem Ratsbürgerentscheid kann die kommunale Vertretungskörperschaft selbst die Initiative ergreifen, um von sich aus an das Gemeindevolk heranzutreten und dieses über eine zuvor definierte Sachfrage entscheiden zu lassen. Aus Gründen der Legitimation und politischen Wertschätzung wird dabei zumeist an das Erfordernis einer qualifizierten Ratsmehrheit angeknüpft. Einen Ratsbürgerentscheid lässt die Gemeindeordnung im Allgemeinen sowie $\$ 26$ GO NW im Besonderen nach jetziger Rechtslage zumindest nach zielbewusster Lesart nicht zu. 


\begin{tabular}{|c|c|c|c|c|c|c|}
\hline & \multicolumn{2}{|c|}{ ja } & \multicolumn{2}{|c|}{ nein } & \multicolumn{2}{|c|}{ weiß nicht } \\
\hline & $\mathrm{n}$ & $\%$ & $\mathrm{n}$ & $\%$ & $\mathrm{n}$ & $\%$ \\
\hline Notwendigkeit einer aufschiebenden Wirkung & 133 & 40,1 & 175 & 52,7 & 24 & 7,2 \\
\hline Kopplung von Abstimmung und Wahl & 203 & 60,4 & 124 & 36,9 & 9 & 2,7 \\
\hline Verankerung eines Ratsbürgerentscheids & 66 & 19,7 & 260 & 77,6 & 9 & 2,7 \\
\hline
\end{tabular}

abnimmt, vermag nun aus dem unmittelbaren Vergleich der strukturell konträrsten Regierungsbezirke Düsseldorf (urban geprägt) und Detmold (ländlich geprägt) wegen eines signifikanten Beurteilungsgefälles die Schlussfolgerung gezogen werden, dass - wenn überhaupt - eher in ländlich geprägten Regionen mit vielen Kleingemeinden eine Affinität für Ratsbürgerentscheide besteht. Jene immerhin 66 Kommunen, die ein Bekenntnis zum Ratsbürgerentscheid abgeben, sind dabei größtenteils der Auffassung, dass daran verschärfte Anforderungen im Sinne einer qualifizierten Mehrheit zur Verfahrensinitiierung zu stellen sind; 77,3 Prozent der einen Ratsbürgerentscheid grundsätzlich befürwortenden Befragten verlangen ausdrücklich eine Zweidrittelmehrheit im Gemeinderat. Anscheinend wird wohl die Gefahr gesehen, dass eine umstrittene politische Entscheidung ansonsten allzu vorschnell auf die Bürger abgewälzt werden könnte.

\subsection{Bewährung von Bürgerbegehren und Bürgerentscheid?}

Unabhängig von der Bewertung einzelner Teilaspekte lässt sich eine vorläufige Gesamtwürdigung der Partizipationsinstrumente unter die Fragestellung „Hat sich die Einführung von Bürgerbegehren und Bürgerentscheid in die Gemeindeordnung Ihres Erachtens bewährt?" fassen ${ }^{24}$. Ein Fünftel der Gemeinden vertritt die Meinung, dass sich die gesetzliche Implementierung „voll und ganz" bewährt habe (60 Kommunen; 19,2 Prozent). Weitere rund 52 Prozent (161 Kommunen) teilen diese Sichtweise mit gewissen Einschränkungen. Demgegenüber ist jeder vierte Befragte (82 Kommunen; 26,3 Prozent) eher weniger von der Praxisbewährung überzeugt, neun Kommunen (2,9 Prozent) sogar überhaupt nicht. Damit ist insgesamt eine recht positive Wertschätzung zugunsten kommunaler Direktdemokratie vorherrschend. Im Vergleich der Gemeindegrößen ist nur jede zehnte kreisfreie Stadt in vollem Umfang überzeugt gegenüber jeder vierten Mittleren kreisangehörigen Stadt. Unter Hinzuziehung der ersten Differenzierungsstufe ergibt sich außerdem, dass die Großen kreisangehörigen Städte mit 77 Prozent an der Bewertungsspitze stehen.

24 Bei den Antwortmöglichkeiten ist eine Präzisierung vorgenommen worden, indem die konträren Vorgaben „ja“ und „nein“ jeweils in ein Mehr und in ein Weniger an Zustimmung respektive Ablehnung eingebettet wurden. Genau an dieser Stelle liegen im Übrigen die Schnittpunkte zu vergleichbaren Umfragen, bei denen zwar Fragestellungen zur Praxisbewährung formuliert werden, aber interessanterweise nicht zur konkreten Ausgestaltung der darauf fußenden Gesetzesgrundlage. 
Hinsichtlich der Begründungen wird ein breites Spektrum abgedeckt, das von der „Bereicherung für die politische Willensbildung“ über die „zunehmende Vereinnahmung durch Parteien und Wählergruppen“ bis zum Vorwurf des „Populismus“ reicht $^{25}$. Interessant ist insbesondere, dass ungeachtet des Kontinuums unterschiedlicher Gewichtungen ein nicht unerheblicher Teil der geäußerten Vorbehalte sich auch auf die für unzureichend empfundene Ausgestaltung der Formen bürgerschaftlicher Mitwirkung beziehen. Die den kommunalen Praxiserfahrungen Rechnung tragende Trennung zwischen Kommunen mit und ohne Bürgerentscheid zum Zeitpunkt der Befragung ergibt des Weiteren, dass die vorbelasteten Gemeinden eine sehr viel positivere Bilanz ziehen als jene ohne absolviertes Referendum ${ }^{26}$.

Damit hat der Bürgerentscheid die kommunale Selbstverwaltung nach Einschätzung derjenigen Städte und Gemeinden, die ihn bereits angewandt haben, zweifellos bereichert, woraus gefolgert werden kann, dass der Großteil dieser Kommunen in der Wahrnehmung dieses Instruments eine nicht nur legale, sondern zugleich legitime punktuelle Intervention erkennt. Direkte Demokratie verfügt demzufolge über ein beachtliches affektives Legitimationsreservoir; die Inanspruchnahme unmittelbar-demokratischer Handlungsoptionen lässt auf eine vorzugsweise positive Resonanz schließen, die nicht ausschließlich auf die Initiatoren eines Bürgerbegehrens und deren Unterstützer beschränkt ist. Diese Erkenntnis gilt umso mehr, als gerade die Verwaltungen bislang in ein festes Entscheidungssystem eingebunden und weitestgehend auf den Gemeinderat als zentrales Entscheidungsgremium fixiert waren. Diese Bindung wird durch den Bürgerentscheid nicht gekappt, vielmehr findet eine davon unabhängige zielbewusste Rückkopplung an das Gemeindevolk selbst statt.

\section{Fazit: Wachsende Toleranz gegenüber direktdemokratischen Optionen}

Für eine abschließende Bewertung der Kommunalumfrage sind mehrere Faktoren ausschlaggebend. Insgesamt scheinen die Beharrungskräfte der Gemeinden sehr ausgeprägt zu sein, da die Positionen stark an der geltenden Rechtslage ausgerichtet sind. ${ }^{27}$ Dies zeigt sich vor allem daran, dass unlängst installierte Verfahrenselemente nicht nur weitgehend akzeptiert, sondern zugleich mit eindrucksvollen Voten bestätigt werden. Die im Ländervergleich keineswegs selbstverständliche (explizite) Beratung durch die Verwal-

25 Vgl. den Streifzug durch die Kommentare, Jens Kösters, a.a.O., S. 188 ff.

26 In der Einzelübersicht (mit Bürgerentscheid / ohne Bürgerentscheid): ,ja, voll und ganz “ (25 / 17 Prozent), „ja, mit Einschränkungen“ (58 / 50 Prozent), „nein, eher weniger“ (17 / 29 Prozent), „nein, ganz und gar nicht" (0 / 4 Prozent).

27 Am 23. Januar 2007 hat die nordrhein-westfälische Landesregierung die Absicht des Innenministers gebilligt, zur geplanten Reform der Gemeindeordnung die so genannte Verbändeanhörung einzuleiten. Die Koalitionsfraktionen von CDU und FDP haben sich auf zukünftige Leitlinien verständigt, die - als Gesamtkonzept betrachtet - kaum Attraktivität ausstrahlen und zudem auf enorme Widerstände, nicht nur von oppositioneller Seite aus, stoßen werden. Der vorgelegte Referentenentwurf widmet sich auch der Frage, inwieweit die demokratische Beteiligung der Bürger gestärkt werden kann; diesem Anspruch soll unter anderem durch die Einführung des Ratsbürgerentscheides Rechnung getragen werden. Das Ergebnis des Reformprozesses bleibt abzuwarten. 
tung, welche auf Antrag zu erteilen ist, kann hierfür stellvertretend als Beispiel genannt werden. Auch an den übrigen Merkmalen des geltenden Regelungskorpus ist zumeist klar ablesbar, dass sich die kommunale Selbstverwaltung erkennbar mit dem Status quo arrangiert hat. Dieses Fazit ist deshalb keineswegs verwunderlich, weil dadurch der Vorrang der Rechtsanwendungskultur gegenüber der reinen Rechtserlasskultur zum Ausdruck kommt und gestärkt wird. Den Städten und Gemeinden muss der Eingewöhnungsprozess gerade auch in sensiblen Politikbereichen zugestanden werden, denn er ist die unabdingbare Voraussetzung dafür, dass sich überhaupt ein nachhaltiges Verständnis für bürgerschaftliches Engagement entfalten kann. Vor diesem Hintergrund ist dem Gesetzgeber unmissverständlich zu signalisieren, Änderungen an der Gemeindeordnung nur nach reiflicher Überlegung vorzunehmen, womit allerdings nicht unterstellt sei, dass dieser banale Grundsatz in der Vergangenheit sträflich missachtet wurde.

Gewisse Fragestellungen entwickeln ferner eine Eigendynamik, zumal sie aufgrund ihrer naturgegebenen Komplexität differenziert zu betrachten sind. Die Reichweite des Kostendeckungsvorschlags ist diesbezüglich ein passendes Beispiel: Eine schlichte $\mathrm{Zu}$ stimmung oder Ablehnung reicht hier nicht aus, vielmehr müssen Ansatzpunkte entwickelt werden, die sowohl für potentielle Antragsteller als auch für die Gemeinde - also Rat und Verwaltung gleichermaßen - tragbar sind. Weil die Ausarbeitung eines Kostendeckungsvorschlags den Initiatoren eines Bürgerbegehrens regelmäßig insofern große Schwierigkeiten bereitet, als zu wenig Klarheit über die im Einzelfall vermeintlich unüberwindbaren Anforderungen besteht, und weil auch die Gemeinden unsicher sein können, was die Rechtskonformität einer bestimmten Maßnahme anbelangt, ist eine sehr behutsame Herangehensweise an diesen Passus erforderlich. Ähnlich verhält es sich mit den Quoren, deren schlichte Existenz respektive konkrete Austarierung wiederholt Gegenstand kritischer Stimmen war.

Bei der Datenaufschlüsselung nach Gemeindegrößenklassen ist an mehreren Stellen deutlich aufgefallen, dass vor allem die kreisfreien Städte „ihren eigenen Kopf“ haben und gewisse Standpunkte noch nachdrücklicher vertreten, etwa was die aufschiebende Wirkung beim Bürgerbegehren betrifft. Diese Ergebnisse eignen sich allerdings nicht dazu, die aufgrund ihrer Einwohnergröße zwangsläufig verschiedenen Städtetypen gegeneinander auszuspielen, zumal die Gemeindeordnung für alle Kommunen NordrheinWestfalens gemeinsame - und identische - Handlungsgrundlage ist und bleiben soll.

Im Hinblick auf bislang ungeklärte, gleichwohl Verbindlichkeit beanspruchende Verfahrensfragen kristallisieren sich einige hoffnungsvolle Ansätze heraus ${ }^{28}$, etwa bei der im Einzelfall möglichen Zusammenlegung von Abstimmungs- und Wahltermin. Damit das mehrheitlich signalisierte Einvernehmen nicht zum bloßen Lippenbekenntnis degradiert wird, ist es an der Zeit, einen verpflichtenden Passus zu formulieren - entweder unmittelbar in der Gemeindeordnung oder aber im Rahmen des individuellen Satzungserlasses. Die Stadt Dortmund hat in dieser Richtung die maßgeblichen Impulse gesetzt, welche uneingeschränkt zur Nachahmung empfohlen sind ${ }^{29}$. Mehrere Kommunen sind inzwischen in die Fußstapfen Dortmunds getreten und stellen damit unter Beweis, dass bür-

28 Entgegen den gewachsenen Strukturen des $₫ 26$ GO NW sind die jeweiligen Mehrheitsmeinungen hier zwar grundsätzlich schwieriger ableitbar, gleichwohl gelingt dieses Vorhaben unter Einbeziehung der vorgetragenen Argumente.

$29 \mathrm{Vgl}$. Jens Kösters, a.a.O., S. 110 f. 
gerschaftliches Engagement kein Gegenstand von „Sonntagsreden“ bleiben muss, sondern auch in direkter Konfrontation mit der „offiziellen Gemeindepolitik“ ein diskutierfähiger Gegenstand ist, dem es mit Respekt zu begegnen gilt.

Ein harmonischer Abschluss der Befragung wird durch die Frage nach der Praxisbewährung erzielt; sie verdeutlicht, dass direkte Demokratie mit der Repräsentativstruktur der Kommunalpolitik verträglich ist, weil der punktuelle Eingriff keine Außerkraftsetzung eines allgemein bewährten Systems zur Folge hat: „Die konzeptionelle Gesamtverantwortung und die Wahrung der Kontinuität kommunaler Politik liegt nach wie vor beim Rat. "30 Die meisten Kommentare der Gemeinden weisen in die Richtung einer sukzessiv wachsenden Toleranz gegenüber direktdemokratischen Handlungsoptionen ${ }^{31}$. Für künftige empirische Befragungen empfiehlt es sich im Übrigen generell, neben dem starren Ankreuzen vorformulierter Antwortalternativen eine kommentierende Begleitung zuzulassen und die gesammelten Einzelmeinungen so zu verdichten, dass sich daraus Argumentationslinien offenbaren können.

30 Fritz Ossenbühl, a.a.O., S. 253.

31 Die Ausgangsfrage ist insoweit dahingehend zu beantworten, dass eine legitime und auch gewollte Bereicherung der Lokalpolitik stattfindet.

\title{
Köpfe statt Themen? Köpfe und Themen! Die Personalisierung der Wahlkampfberichterstattung in Deutschland und in den USA*
}

\author{
Kim Jucknat
}

Die Personalisierung der Politik ist nicht erst seit heute ein zentrales Thema der Wahlkampfkommunikationsforschung ${ }^{1}$. Der frühere Wahlkampfmanager der CDU Peter Radunski bemerkte schon Anfang der achtziger Jahre: „Politische Führer als Verkörperung politischer Ideale hat es zu allen Zeiten gegeben." 2 Betrachtet man den Wahlwerbeslogan der CDU beim Bundestagswahlkampf 1969 „Auf den Kanzler kommt es an“ oder etwa

* Der Beitrag basiert auf der unveröffentlichten Magisterarbeit aus dem Jahr 2005 mit dem Titel „Politikvermittlung in parlamentarischen und präsidentiellen Demokratien“, Universität Mannheim, Lehrstuhl für Politische Wissenschaft und International Vergleichende Sozialforschung Prof. Dr. Jan van Deth; Datensatz und Manuskript können auf Anfrage eingesehen werden.

1 Vgl. etwa Helmut Norpoth, Kanzlerkandidaten, in: PVS, 18. Jg. (1977), S. 551 - 572; Max Kaase, Is there personalization in politics? Candidates and Voting Behaviour in Germany, in: International Political Science Review, 15. Jg. (1994), S. 211 - 230; Peter Radunski, Wahlkämpfe. Moderne Wahlkampfführung als politische Kommunikation, München 1980; Klaus Schönbach / Holli Semetko, Medienberichterstattung und Parteienwerbung im Bundestagswahlkampf 1990, in: Media Perspektiven, 7. Jg. (1994), S. 328 - 340.

2 Peter Radunski, a.a.O., S. 15. 